\title{
Aumento de Resolução Temporal de Sequências de Imagens do Trato Vocal por meio de Registro das Imagens
}

\author{
Ana L. D. Martins1 \\ Nelson D. A. Mascarenhas1 \\ Cláudio A. T. Suazo2
}

Resumo: O imageamento por ressonância magnética tem sido bastante utilizado no estudo da produção da fala. Sequências de imagens do trato vocal adquiridas durante a emissão de palavras e fonemas permitem a identificação dinâmica das formas assumidas por este tubo acústico. Entretanto, é importante ressaltar que as resoluções espacial e temporal necessárias para a identificação do movimento dos articuladores da fala variam de acordo com a velocidade e localidade desse movimento, e tal informação não é conhecida a priori. Abordagens existentes procuram aprimorar a resolução das sequências de imagens melhorando o processo de aquisição ou utilizando meios de aquisição mais potentes, o que pode ser financeiramente inviável. O método proposto neste artigo procura melhorar a resolução temporal por meio de um método de registro não-rígido proposto na literatura. O movimento identificado pelo registro permite o aumento de resolução temporal por meio de uma técnica de interpolação por compensação de movimento. O movimento presente em toda a sequência é considerado na geração de cada imagem intermediária. Dessa forma, o movimento dos articuladores da fala nessas imagens é coerente com o

\footnotetext{
1 Departamento de Computação, UFSCar, CP 676, CEP: 13.565-905, São Carlos, SP \{ana_martins, nelson edc.ufscar.br\}

2 Departamento de Engenharia Química, UFSCar, CP 676, CEP: 13.565-905, São Carlos, SP \{claudio@power.ufscar.br\}
} 
Aumento de Resolução Temporal de Sequências de Imagens do Trato Vocal por meio de Registro das Imagens

movimento presente em toda a sequência. Os resultados indicam a eficiência do método proposto.

\begin{abstract}
Dynamic magnetic resonance imaging (MRI) is an emerging technique for studying speech production. In general, vocal tract image sequences are acquired during the speech of a word or phoneme. These sequences allow the identification of shapes taken by the vocal tract during speech production. However, there is no prior knowledge about the spatial and temporal resolution requirements, which are expected to vary for different speech tasks. Available approaches try to enhance the resolution of the images by empowering the acquisition devices, which can be expensive. In this paper, we propose an alternative approach to enhance temporal resolution based solely on the observed image sequences. Our continuous motion compensated interpolation (MCI) method uses a previous non-rigid image registration method, which provides an intuitive background for temporal resolution enhancement. We consider motion along the whole sequence instead of only the enclosing neighbors. Therefore the speech articulators movement is more accurately represented. Results indicate the effectiveness of our approach.
\end{abstract}


Aumento de Resolução Temporal de Sequências de Imagens do Trato Vocal por meio de Registro das

Imagens

\section{Introdução}

De acordo com Bresch et al. [1], diversas áreas de pesquisa, como a engenharia e a linguística, tem grande interesse no conhecimento detalhado a respeito da produção da fala. Esse conhecimento permite a modelagem do sinal da fala, o que pode ser explorado no desenvolvimento de sistemas de reconhecimento, codificação e síntese da mesma. Além disso, pesquisas podem ser conduzidas para solucionar questões dentro da fonética, como variabilidades e desordens na produção da fala.

O trato vocal pode ser visto como um tubo acústico com área da seção transversal variável. Primeiramente, dados a respeito da forma do trato vocal eram coletados por meio de fontes radiográficas. Entretanto, o imageamento por raios-X apresenta uma série de limitações. Além do risco de exposição à radiação ionizante, as áreas das seções transversais são obtidas aplicando transformações nas larguras medidas através das projeções laterais. Assim, chega-se a apenas a uma aproximação da morfologia do trato vocal. De acordo com Baer et al. [2], o imageamento por ressonância magnética (MRI - Magnetic Resonance Imaging), é o único meio que fornece dados tridimensionais detalhados do trato vocal sem efeitos danosos à pessoa. Entretanto, é importante ressaltar que as resoluções espacial e temporal necessárias para a identificação do movimento dos articuladores da fala variam de acordo com a velocidade e a área afetada por esse movimento, e tal informação não é conhecida a priori. Além disso, segundo Narayanan et al. [3], mesmo que os avanços em MRI tenham possibilitado uma melhora na qualidade da informação a respeito da trajetória dos articuladores da fala, essa técnica de imageamento ainda não possui a resolução temporal necessária para captar as características dinâmicas do movimento da língua. Abordagens existentes procuram melhorar a resolução das sequências de imagens melhorando o processo de aquisição ou utilizando meios de aquisição mais potentes, o que pode ser financeiramente inviável. Dessa forma, o desenvolvimento de técnicas de aumento de resolução temporal de sequências de imagens observadas, por meio de técnicas de processamento de imagens digitais, é de grande interesse nesse contexto.

O registro de imagens tenta identificar a transformação geométrica ótima entre dados correspondentes em duas imagens [4]. Essa transformação pode ser utilizada para quantificar modificações entre as imagens. Nesse caso, além de identificar a transformação que mapeia pontos de uma imagem nos pontos correspondentes na outra imagem, o registro também descreve o movimento e as deformações que ocorreram entre as imagens. Dessa forma, o registro pode ser utilizado para o aumento de resolução temporal de uma sequência de imagens. Imagens intermediárias às imagens observadas podem ser geradas respeitando o movimento e deformação existente na sequência.

Nesse contexto, a abordagem proposta utiliza um método de registro previamente proposto na literatura [6] para alcançar o aumento de resolução temporal de sequências de imagens de ressonância magnética (MR - Magnetic Resonance) do trato vocal humano. Esse método descreve a transformação entre pares de imagens por meio de free-form deformations 
(FFD) e interpolação B-spline em malhas de pontos de controle uniformemente espaçados. Modelando a transformação dessa forma, seus únicos parâmetros são as coordenadas dos pontos de controle da malha. Assim, considerando as malhas correspondentes a duas imagens adjacentes, uma imagem intermediária pode ser gerada construindo uma malha, na qual os pontos de controle possuem coordenadas intermediárias às coordenadas presentes nas malhas das imagens observadas. Além disso, a fim de gerar imagens intermediárias que conservem o movimento presente em toda a sequência, as malhas de pontos de controle intermediárias são geradas considerando as coordenadas dos pontos de controle de toda a sequência observada.

Os resultados mostram que o método de registro adotado é capaz de identificar o movimento e deformações presentes entre as imagens de forma satisfatória. Além disso, as imagens intermediárias geradas pela abordagem de aumento de resolução temporal proposta não apresentam artefatos e são coerentes com o movimento presente em toda a sequência observada.

\section{Registro Não-Rígido de Imagens}

Imagens do trato vocal humano utilizadas em estudos de síntese articulatória apresentam deformações semelhantes às ilustradas na Figura 1. O erro entre as duas imagens, apresentado na Figura 1(c), enfatiza a presença de deformações concentradas na região da boca. Dessa forma, é necessário um método de registro não-rígido para identificar o movimento presente nas sequências observadas.

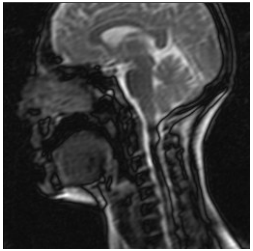

(a)

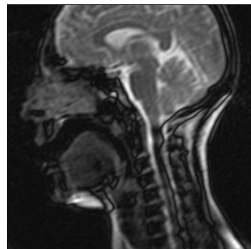

(b)

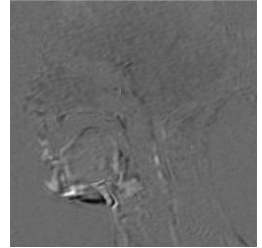

(c)

Figura 1. Dois momentos a emissão da fala de uma palavra e o erro entre as duas imagens.

\subsection{FFD, interpolação B-spline e malhas de pontos de controle}

Rueckert et al. [6] apresentou um método de registro não-rígido que descreve a transformação entre pares de imagens por meio de FFD e interpolação B-spline em malhas de pontos de controle uniformemente espaçados. Introduzidas por Sederberg and Parry [7], FFD é uma abordagem utilizada em aplicações de computação gráfica para modelar objetos tridimensionais deformáveis. O objeto é deformado através da manipulação de pontos de 
controle. Considerando um caso bidimensional, a Figura 2 mostra a deformação de uma imagem devido à manipulação da malha de pontos de controle uniformemente espaçados mostrada na Figura 2(b).

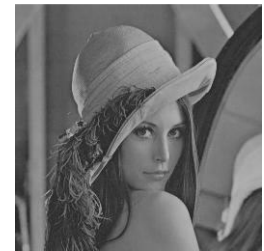

(a)

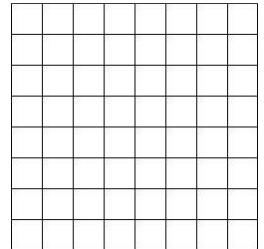

(b)

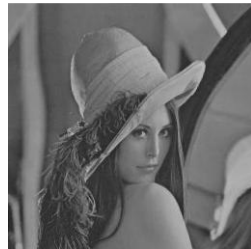

(c)

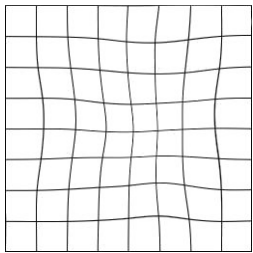

(d)

Figura 2. Deformação da imagem Lena por meio de FFD baseadas em interpolação B-spline.

A abordagem proposta por Rueckert et al. modela as deformações por uma transformação, a qual combina movimento global da cena e deformações locais $\varphi(x, y)=\varphi_{\text {global }}(x, y)+\varphi_{\text {local }}(x, y) . \varphi_{\text {global }}$ modela uma transformação afim aplicada a toda a imagem

$$
\varphi_{\text {global }}(x, y)=\left[\begin{array}{ll}
\theta_{11} & \theta_{12} \\
\theta_{21} & \theta_{22}
\end{array}\right] \cdot\left[\begin{array}{l}
x \\
y
\end{array}\right]+\left[\begin{array}{l}
\theta_{13} \\
\theta_{23}
\end{array}\right],
$$

sendo $\Theta=\left\{\theta_{i j} \mid 1 \leq i \leq 2,1 \leq j \leq 3\right\}$ os parâmetros dessa transformação. As deformações locais são modeladas por FFDs baseadas em interpolação B-spline em uma malha de pontos de controle. Considerando $\Omega=\{(x, y) \mid 0 \leq x \leq X, 0 \leq y \leq Y\}$ o suporte da imagem, e $\Phi$ a malha de pontos de controle, $n_{x} \times n_{y}$, com espaçamento uniforme $\delta, \varphi_{\text {local }}$ é dado por

$$
\varphi_{\text {local }}(x, y)=\sum_{m=0}^{3} \sum_{n=0}^{3} B_{m}(u) B_{n}(v) \phi_{i+m, j+n},
$$

onde $i=\left\lfloor x / n_{x}\right\rfloor, j=\left\lfloor y / n_{y}\right\rfloor, u=x / n_{x}-\left\lfloor x / n_{x}\right\rfloor, v=y / n_{y}-\left\lfloor y / n_{y}\right\rfloor$ e $B_{l}$ é a $l$ ésima função B-spline unidimensional 


$$
\begin{aligned}
& B_{0}(u)=(1-u)^{3} / 6 \\
& B_{1}(u)=\left(3 u^{3}-6 u^{2}+4\right) / 6 \\
& B_{2}(u)=\left(-3 u^{3}+3 u^{2}+3 u+1\right) / 6 \\
& B_{3}(u)=u^{3} / 6 .
\end{aligned},
$$

Dessa forma, as coordenadas dos pontos de controle $\phi_{i, j}$ são os parâmetros dessa transformação. Na abordagem proposto por Rueckert et al. sua identificação é automática, não dependendo do conhecimento de um especialista ou de características previamente identificadas na imagem. Apesar de existirem abordagens sofisticadas para a identificação automática de pontos de controle para a aplicação do registro, esse ainda é um processo complicado e não é completamente automático. Além disso, funções base B-spline cúbicas possuem suporte finito. Assim, cada ponto de controle afeta apenas sua vizinhança mais próxima, permitindo que deformações bastante localizadas na cena possam ser modeladas.

A resolução da malha de pontos de controle determina o quão localizada pode ser a deformação não-rígida. Resoluções mais baixas permitem apenas deformações mais globais. Por outro lado, resoluções mais finas permitem deformações localizadas em pequenas regiões da imagem. Assim, Lee et al. [9] propuseram uma FFD multi-nível na qual a resolução da malha de pontos de controle vai sendo refinada para satisfazer exatamente os requisitos da deformação existente entre as imagens e conseguir o melhor compromisso entre o grau da deformação e o custo computacional. Considere $\Phi_{0}, \ldots, \Phi_{L}$ uma hierarquia de malhas de pontos de controle na qual o espaçamento entre os pontos diminui de $\Phi_{l}$ para $\Phi_{l+1}$. Cada malha $\Phi_{l}$ e a respectiva FFD define uma transformação local $\varphi_{\text {local }}^{l}$ e a soma de todas essas transformações define $\varphi_{\text {local }}$

$$
\varphi_{\text {local }}(x, y)=\sum_{l=0}^{L} \varphi_{\text {local }}^{l}(x, y) .
$$

Rueckert et al. regularizou a transformação impondo a restrição de suavidade a seguir

$$
C_{\text {suavidade }}(\varphi)=\frac{1}{V} \int_{0}^{X} \int_{0}^{Y}\left(\frac{\partial^{2} \varphi}{\partial x^{2}}\right)^{2}+\left(\frac{\partial^{2} \varphi}{\partial y^{2}}\right)^{2}+2\left(\frac{\partial^{2} \varphi}{\partial x y}\right)^{2} \mathrm{~d} x \mathrm{~d} y .
$$

Além disso, os autores utilizaram a informação mútua normalizada (NMI - Normalized Mutual Information) como medida de similaridade entre as imagens. Considerando a 
imagem de referência $R$, e uma imagem $T$ a ser registrada com $R$, a NMI entre $R$ e a imagem transformada $T_{\varphi}$ é dada por

$$
C_{\text {similaridade }}\left(R, T_{\varphi}\right)=\frac{H(R)+H\left(T_{\varphi}\right)}{H\left(R, T_{\varphi}\right)},
$$

onde $H(R)$ e $H\left(T_{\varphi}\right)$ são as entropias marginais de $R$ e $T_{\varphi}$, e $H\left(R, T_{\varphi}\right)$ é o histograma conjunto de $R$ e $T_{\varphi}$ [10]. Nesse contexto, os parâmetros da transformação ótima, $\Theta$ e $\Phi$, são encontrados minimizando uma função custo formada por uma composição de $C_{\text {suavidade }}$ e $C_{\text {similaridade }}$

$$
C(\Theta, \Phi)=-C_{\text {similaridade }}\left(R, T_{\varphi}\right)+\lambda C_{\text {suavidade }}(\varphi),
$$

onde $\lambda$ é um parâmetro que modela o compromisso entre o alinhamento entre as duas imagens e a suavidade da transformação. A otimização é feita por uma técnica iterativa de gradiente descendente com passo na direção do vetor gradiente, de tamanho $\mu$. O algoritmo para se um ótimo local da função custo foi encontrado $(\|\nabla C\| \leq \varepsilon$, para $\mathcal{\varepsilon}$ pequeno e positivo).

\section{Método Proposto}

O método de registro não-rígido apresentado por Rueckert et al. foi capaz de modelar adequadamente as deformações existentes entre as imagens observadas, como evidenciado na Figura 3. Considerando a imagem mostrada na Figura 3(a) como imagem de referência, a malha de pontos de controle correspondente é formada por pontos uniformemente espaçados como mostrado na Figura 3(b) (os pontos de controle estão localizados nas intersecções das linhas pretas). A transformação que leva a imagem de referência na imagem mostrada na Figura 3(c) é modelada pela malha de pontos de controle mostrada na Figura 3(d). As Figuras 3(e) e 3(f) mostram a imagem de referência após a transformação identificada pelo método de registro, e o erro entre as imagens 8(c) e 8(e), respectivamente. Como se pode notar, as imagens são muito próximas. É importante ressaltar que as imagens com as malhas de pontos de controle são apenas ilustrativas, não sendo necessárias para a execução do método de registro e do aumento de resolução temporal da sequência. 
Aumento de Resolução Temporal de Sequências de Imagens do Trato Vocal por meio de Registro das Imagens

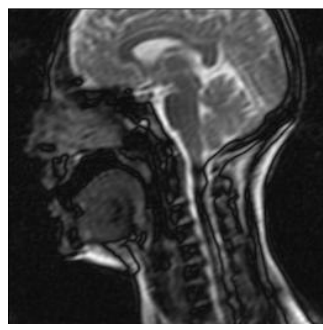

(a)

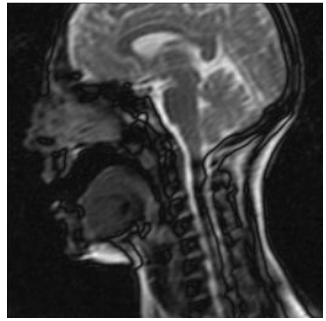

(c)

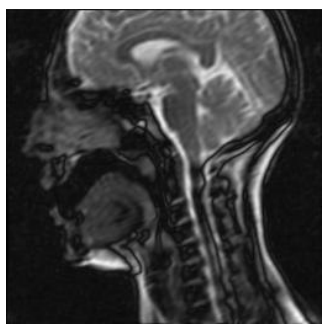

(e)

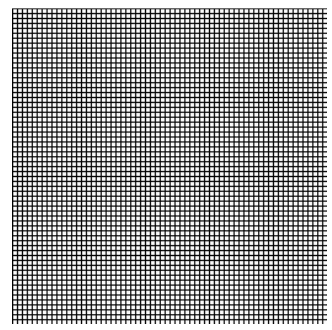

(b)

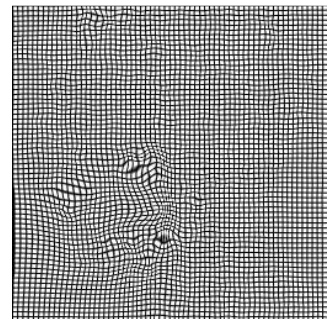

(d)

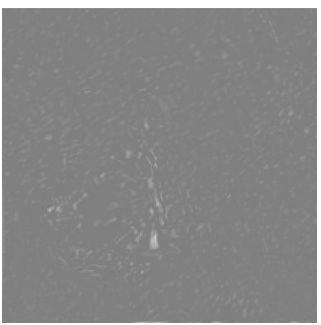

(f)

Figura 3. (a)-(d) Identificação das deformações presentes entre duas imagens, (e) Imagem Registrada e (f) Erro entre as imagens (c) e (e).

Afim de aumentar a resolução temporal de sequências de imagens de um dado evento, abordagens existentes utilizam múltiplas fontes de aquisição ou várias aquisições da mesma fonte com um pequeno atraso entre elas[12][13]. Entretanto, quando se possui apenas uma única aquisição de um evento, o aumento de resolução é alcançado por meio de interpolação entre as observações. Uma abordagem simples seria combinar as intensidades de mesma localização espacial de dois frames adjacentes para gerar um frame intermediário. Essa abordagem apresenta qualidade visual aceitável na ausência de movimento, mas ela causa borramento em partes que se movem de uma imagem para outra. Técnicas de interpolação baseadas em movimento são capazes de reduzir esses artefatos. Penney et al. [14] apresenta um método de interpolação entre fatias adjacentes de um conjunto de dados tomográficos 
tridimensional. Fatias intermediárias às fatias existentes foram geradas a fim de alcançar uma dimensão isotrópica dos voxels da imagem tridimensional. O método se baseia na correspondência espacial entre as fatias, identificada pelo método de registro não-rígido apresentado por Rueckert et al. De acordo com os autores, para que seja possível aplicar a interpolação baseada no registro assume-se duas hipóteses: imagens adjacentes possuem características semelhantes, e o algoritmo de registro é capaz de encontrar uma transformação que mapeia tais características de uma imagens nas características correspondentes na outra imagem. No caso das sequências de imagens de MR do trato vocal, as duas hipóteses são verdadeiras e, dessa forma, é possível gerar imagens intermediárias de acordo com as transformações identificadas pelo método de registro. Entretanto, como ilustrado na Figura 4, Penney et al. aplicaram interpolação linear na direção encontrada pelo algoritmo de registro para identificar a posição de pontos correspondentes na fatia intermediária. No contexto das imagens utilizadas neste projeto, essa pode não ser a melhor abordagem. Uma sequência de imagens retrata o movimento dos articuladores da fala. Dessa forma, por se tratar de um movimento contínuo e suave, acredita-se que a interpolação linear na direção do vetor de movimento não seja a mais adequada. Além disso, o movimento presente em imagens intermediárias deve ser coerente com o movimento presente em toda a sequência. Isso pode ser garantido se forem consideradas mais imagens na geração do frame intermediário. Segundo Thévenaz et al. [15], existem várias motivações para o uso de splines ou funções baseadas em splines na interpolação. Essas funções são capazes de evitar a presença de artefatos, apresentando um ótimo compromisso entre qualidade e custo computacional. Nesse contexto, propõe-se que o aumento de resolução temporal de uma sequência de imagens de MR do trato vocal seja feito por meio de interpolação por splines cúbicas como ilustrado na Figura 5.

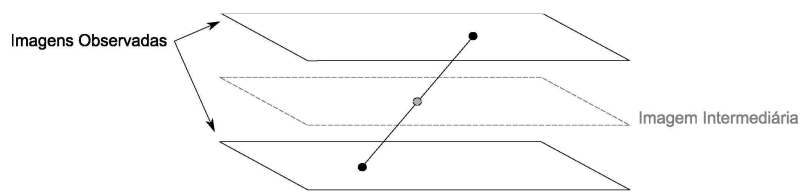


Aumento de Resolução Temporal de Sequências de Imagens do Trato Vocal por meio de Registro das Imagens

Figura 4. Ilustração da interpolação entre fatias adjacentes (adaptado de [14]).

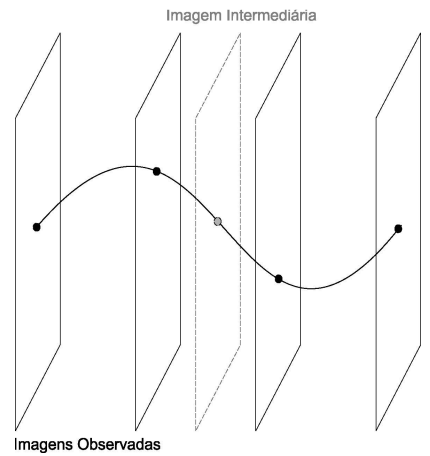

Figura 5. Ilustração da interpolação entre duas imagens por meio de funções spline cúbicas.

A representação por meio de malhas de pontos de controle se mostrou uma ferramenta poderosa para o aumento de resolução temporal. É importante notar que, de acordo com a correspondência entre as malhas de pontos de controle, imagens intermediárias às imagens observadas podem ser geradas simplesmente posicionando pontos de controle em posições coerentes (Equação 3). Considerando uma sequência de imagens do trato vocal $I_{0}, \ldots, I_{k}$, $k \in \mathrm{N}$, o algoritmo de registro foi aplicado a cada par de imagens consecutivas como ilustrado na Figura 6. Dessa forma, encontradas as malhas de pontos de controle, é possível identificar a transformação entre qualquer par de imagens nessa sequência. $O$ registro é aplicado a cada par de imagens consecutivas porque entre imagens muito distantes as deformações podem ser grandes demais e, por isso, não serem facilmente capturadas.

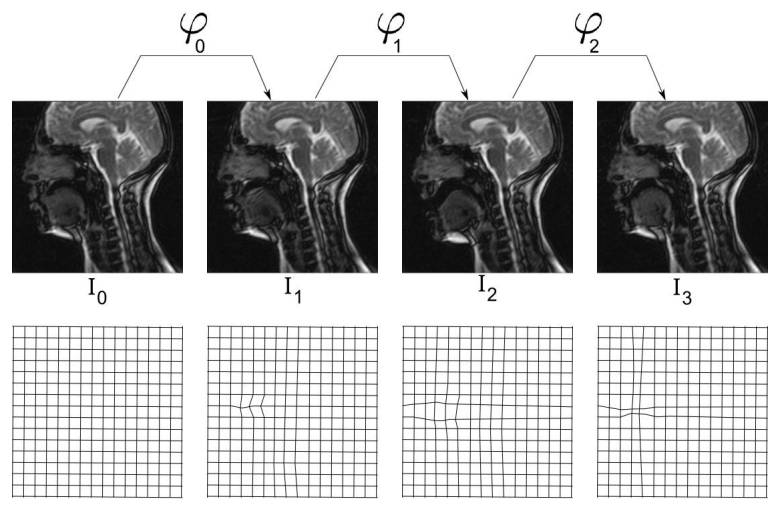


Figura 6. Ilustração da aplicação do método de registro a cada par de imagens consecutivas.

A malha de pontos de controle correspondente a uma imagem intermediária é gerada da seguinte forma. Sejam $\phi_{i, j}^{1}, \ldots, \phi_{i, j}^{k}$ as coordenadas dos pontos de controle correspondentes, de índice $(i, j)$, das imagens observadas $I_{0}, \ldots, I_{k}$. As coordenadas do ponto de controle correspondente em uma imagem intermediária são identificadas por interpolação por splines cúbicas das coordenadas $\left[\phi_{i, j}^{1}, \ldots, \phi_{i, j}^{k}\right]$, como ilustrado na Figura 7 . Isso foi implementado utilizando a função spline() do MatLab.

A Figura 8 mostra o movimento dos pontos de controle $\phi_{1,1}^{n}, n=1, \ldots, 20$, de um conjunto de 20 imagens do trato vocal observadas. As Figuras 8(a) e 8(c) mostram o movimento considerando a interpolação por funções spline cúbicas nas direções horizontal e vertical, respectivamente, e as Figuras 8(b) e 8(d) mostram o movimento considerando a interpolação linear nas direções horizontal e vertical, respectivamente. Nota-se que a interpolação por funções spline cúbicas é mais coerente no contexto da modelagem de movimento entre as imagens.

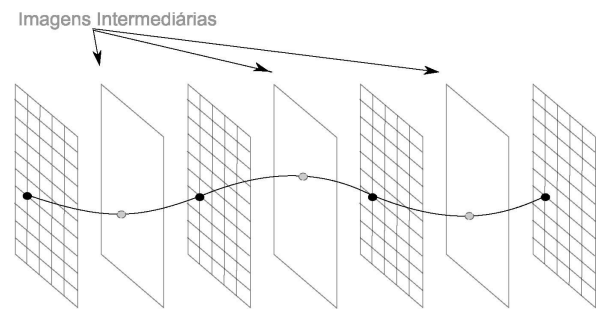

Figura 7. Ilustração da geração de malhas de pontos de controle intermediaries às malhas identificadas pelo método de registro.
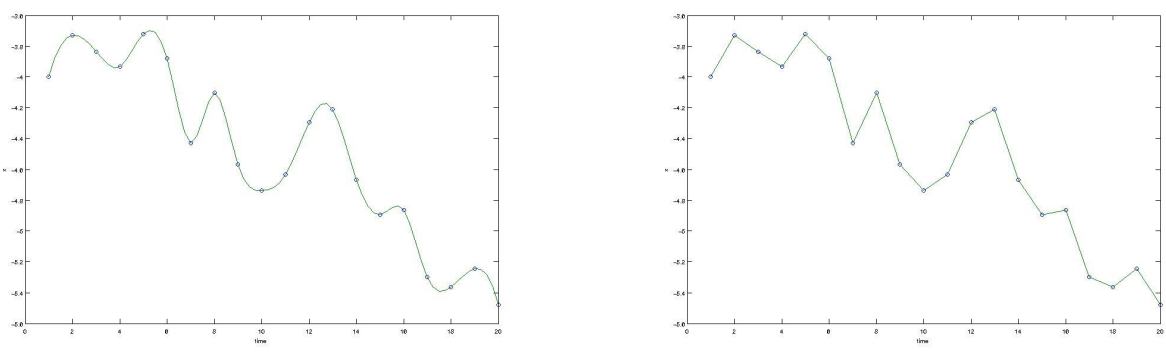
Aumento de Resolução Temporal de Sequências de Imagens do Trato Vocal por meio de Registro das Imagens

(a)

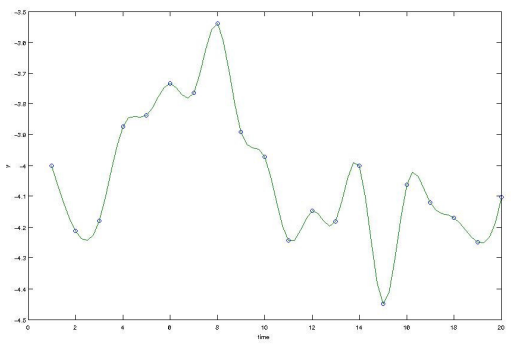

(c) (b)

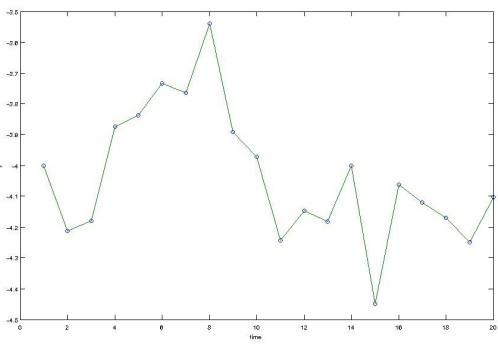

(d)

Figura 7. Movimento dos pontos de controle $\phi_{1,1}^{n}, n=1, \ldots, 20$, de um conjunto de 20 imagens.

Identificada a malha de pontos de controle da imagem intermediária, a transformação referente a essa malha é aplicada nas duas imagens adjacentes como ilustrado na Figura 8. Os pixels da nova imagem são encontrados pela média ponderada dos pixels das imagens adjacentes transformadas, cujos pesos são definidos de acordo com a distância da nova imagem a cada uma de suas vizinhas. A imagem da qual a nova imagem estiver mais próxima recebe um peso mais alto, por exemplo, considerando que duas imagens sejam referentes aos instantes 1 e 2, para gerar a imagem referente ao instante 1.75, os pesos seriam 0.25 para os pixels da imagem no instante 1 e 0.75 para os pixels da imagem no instante 2 (a soma dos pesos sempre é igual a 1). Dessa forma, o aumento de resolução temporal preserva o movimento presente nas imagens observadas.

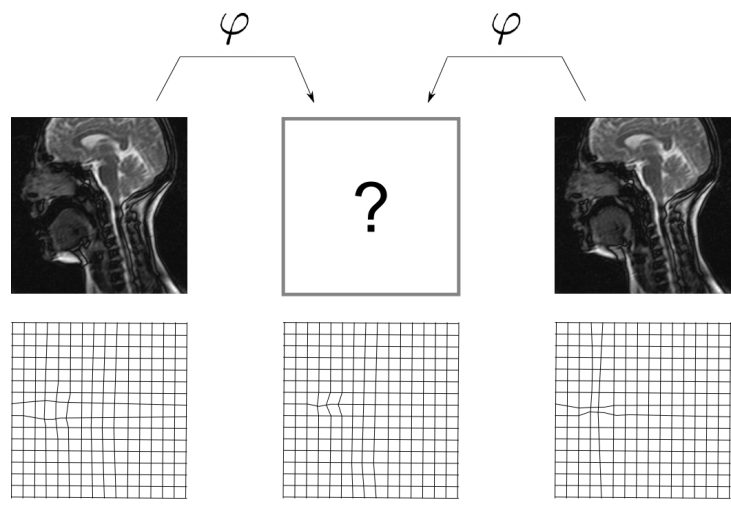


Aumento de Resolução Temporal de Sequências de Imagens do Trato Vocal por meio de Registro das Imagens

Figura 8. Aumento de resolução temporal através da soma ponderada entre as imagens vizinhas transformadas.

\section{Resultados}

Com o intuito de avaliar visualmente a abordagem proposta para o aumento de resolução temporal, considerando sequências de imagens de MR do trato vocal de $256 \times 256$, foram geradas imagens intermediárias às imagens observadas. A sequência mostrada na Figura 9 foi registrada como ilustrado na Figura 6. A abordagem hierárquica de registro não-rígido resultou nas malhas de pontos de controle, com resolução de $67 \times 67$, também apresentadas na Figura 9. É importante notar que a primeira malha_e composta por pontos uniformemente espaçados. Dessa forma, a primeira imagem está sendo considerada como referência. Como é possível observar na Figura 9, o método de registro foi capaz de modelar coerentemente as deformações presentes entre as imagens já que as malhas são condizentes com tais deformações.

A proposta de aumento de resolução temporal baseada nas transformações identificadas pelo método de registro não-rígido, possibilita a geração de infinitas imagens intermediárias às imagens observadas, já que se trata de um modelo contínuo. A Figura 10(b) apresenta uma imagem gerada entre as duas primeiras imagens da sequência mostrada na Figura 9. Considerando que as quatro imagens observadas foram adquiridas nos momentos $t=1, \ldots, 4$, a imagem intermediária foi gerada no momento $t=1,5$. É possível notar que não existem artefatos e, como indicado pela malha gerada, a imagem é coerente com o movimento existente na sequência.
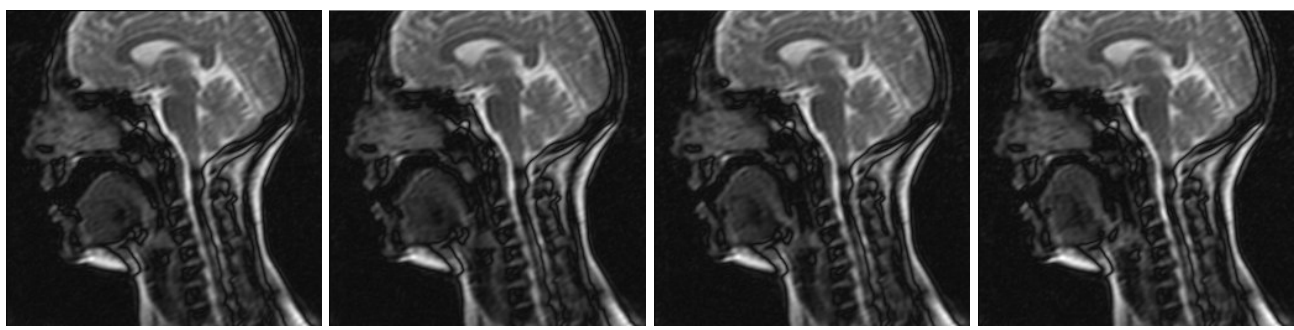
Aumento de Resolução Temporal de Sequências de Imagens do Trato Vocal por meio de Registro das Imagens
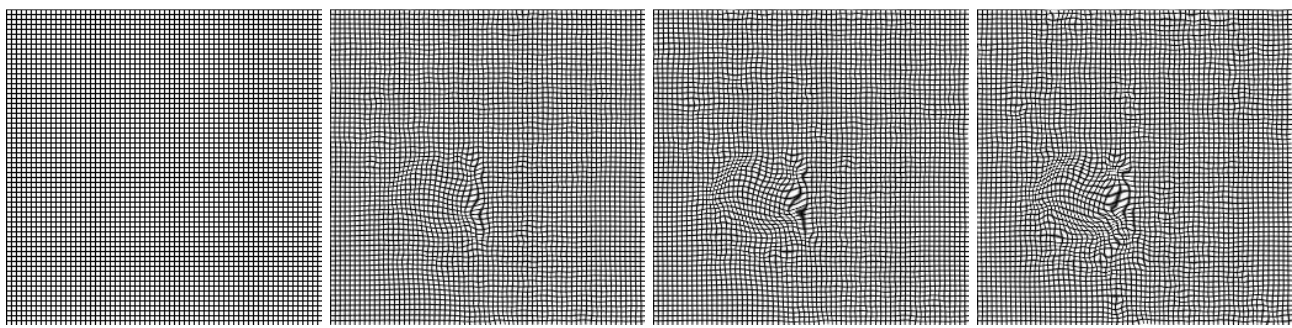

Figura 9. Sequência de imagens do trato vocal e as respectivas malhas de pontos de controle.
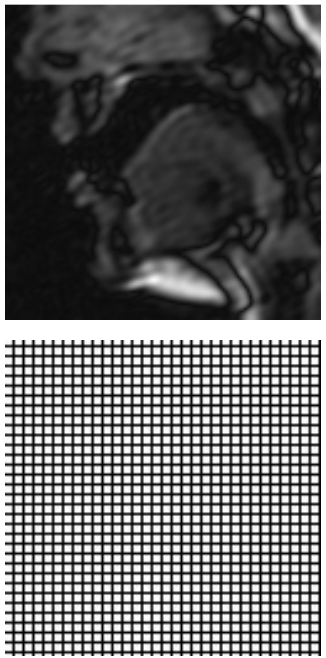

(a)
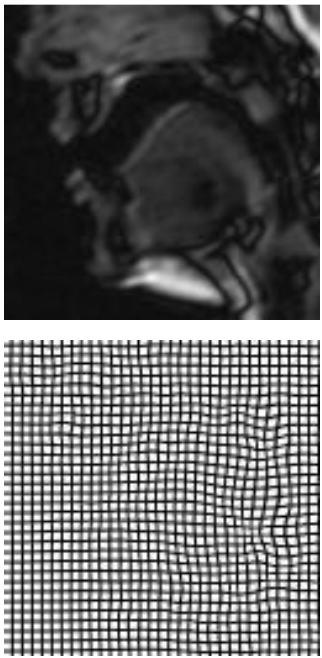

(b)
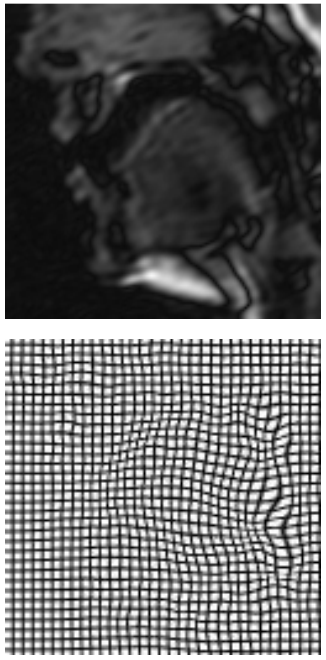

(c)

Figura 10. Detalhe da imagem intermediária (b) gerada entre as duas primeiras imagens da sequência, (a) e (c) respectivamente, e as malhas de controle correspondents.

Para efeitos de comparação, infelizmente não foi encontrado nenhum método de interpolação por compensação de movimento de imagens do trato vocal utilizadas em pesquisas de síntese articulatória. Assim, as imagens geradas pela abordagem proposta foram comparadas com imagens geradas combinando pixels de mesma localização espacial e interpolação linear com base nas transformações identificadas pelo método de registro utilizado pela abordagem proposta. A Figura 11 mostra imagens intermediárias $(t=1,5)$ às duas primeiras imagens da sequência mostrada na Figura 9, geradas pelo método proposto, combinando pixels de mesma localização espacial, e por interpolação linear do vetor de movimento identificado pelo método de registro utilizado neste projeto, respectivamente. Como se pode notar pela figura, a imagem gerada combinando pixels de mesma localização 
espacial ficou borrada nas áreas de maior movimento. As imagens geradas pelo método proposto e por interpolação linear do vetor de movimento estão muito próximas. Entretanto, como discutido na Figura 7, na geração de infinitas imagens entre cada par de imagens observadas, o método proposto modela o movimento de forma mais coerente. Além disso, a etapa de maior custo computacional no aumento de resolução temporal de uma sequência é o registro das imagens. O custo computacional para a geração dos frames intermediários é o mesmo para a interpolação linear do vetor de movimento e para a interpolação proposta.

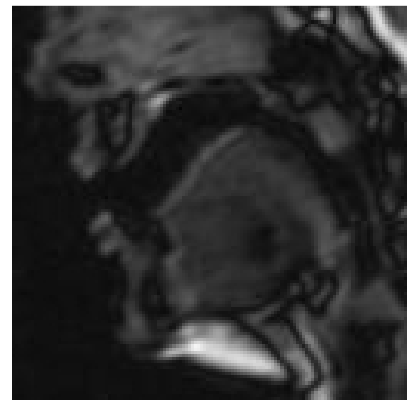

(a)

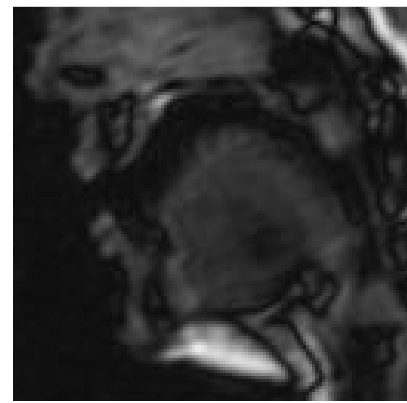

(b)

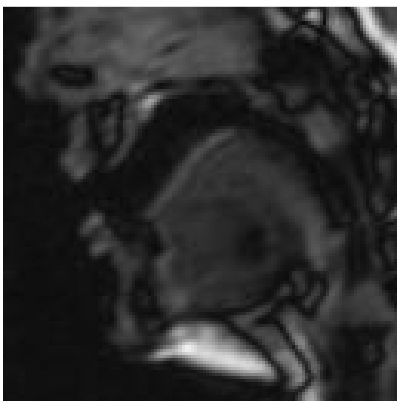

(c)

Figura 11. (a) imagem gerada pelo método proposto, (b) Imagem gerada combinando pixels de mesma localização espacial e (c) Imagem gerada por interpolação linear do vetor vetor de movimento identificado pelo método de registro proposto por Rueckert et al.

\section{Conclusão}

Este projeto discute o aumento de resolução temporal de uma sequência de imagens de RM do trato vocal humano, utilizadas em estudos de síntese articulatória. O método proposto busca melhorar a resolução temporal por meio de um método de registro não-rígido proposto na literatura [6]. O movimento identificado pelo registro permite o aumento de resolução temporal por meio de uma técnica de interpolação por compensação de movimento. O movimento presente em toda a sequência é considerado na geração de cada imagem intermediária. Assim, o movimento é coerente com toda a sequência. Os resultados indicam a eficiência do método proposto.

\section{Agradecimentos}

Agradecemos aos professores Dr. António Joaquim Silva Teixeira e Dr. Augusto Marques Ferreira Silva do Instituto de Engenharia Eletrônica e Telemática de Aveiro (IEETA) da Universidade de Aveiro, Portugal, pelo fornecimento das imagens utilizadas 
Aumento de Resolução Temporal de Sequências de Imagens do Trato Vocal por meio de Registro das Imagens

neste projeto. Essas imagens fazem parte do Projeto HERON - Um Ambiente Computacional para Investigação em Síntese Articulatória do Português, POSI/PLP/57680/2004.

\section{Referências}

[1] E. Bresch, Y.-C. Kim, K. Nayak, D. Byrd, and S. Narayanan. Seeing speech: Capturing vocal tract shaping using realtime magnetic resonance imaging [Exploratory DSP]. Signal Processing Magazine, IEEE, 25(3):123-132, May 2008.

[2] T. Baer, J. C. Gore, L. C. Gracco, and P.W. Nye. Analysis of vocal tract shape and dimensions using magnetic resonance imaging: Vowels. J. Acoust. Soc. Am., 90(2):799-828, Aug 1991.

[3] S. Narayanan, K. Nayak, S. Lee, A. Sethy, and D. Byrd. An approach to real-time magnetic resonance imaging for speech production. The Journal of the Acoustical Society of America, 115(4):1771-1776, 2004.

[4] J. Modersitzki. Numerical Methods for Image Registration. Oxford University Press, 2004.

[6] D. Rueckert, L. I. Sonoda, C. Hayes, D. L. G. Hill, M. O. Leach, and D. J. Hawkes. Nonrigid Registration Using Free-Form Deformations: Application to Breast MR Images. IEEE Transactions on Medical Imaging, 18(8):712-721, 1999.

[7] T. W. Sederberg and S. R. Parry. Free-form deformation of solid geometric models. SIGGRAPH Comput. Graph., 20(4):151-160, 1986.

[9] S. Lee, G. Wolberg, K.-Y. Chwa, and S. Y. Shin. Image Metamorphosis with Scattered Feature Constraints. IEEE Transactions on Visualization and Computer Graphics, 2:337-354, 1996.

[10] Viola, P. A. Alignment by Maximization of Mutual Information. Tese de Doutoramento, Massachusetts Institute of Technology, 1995.

[12] Caspi, Y.; Irani, M. Spatio-Temporal Alignment of Sequences. IEEE Trans. On Pattern Analysis and Machine Intelligence (PAMI), v. 24, n. 11, p. 1409-1424, 2002.

[13] Singh, M.; Basu, A.; Mandal, M. Event Dynamics Based Temporal Registration. Multimedia, IEEE Transactions on, v. 9, n. 5, p. 1004-1015, 2007.

[14] Penney, G. P.; Schnabel, J. A.; Rueckert, D.; Viergever, M. A.; Niessen, W. J. Registration-based interpolation. IEEE Transactions on Medical Imaging, v. 23, n. 7, p. 922-926, 2004. 